CIUDAD Y TERRITORIO

ESTUDIOS TERRITORIALES

ISSN(P): 1133-4762; ISSN(E): 2659-3254

Vol. LII, № 204, verano 2020

Págs. 321-334

https://doi.org/10.37230/CyTET.2020.204.09

CC BY-NC 4.0

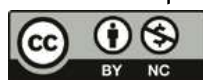

\title{
Transformaciones territoriales por cambios en las redes energéticas. El caso del área portuaria Bahía Blanca Coronel Rosales (Argentina)
}

\author{
Luciana-Vanesa CLEMENTI(1) \\ María-Sofía VilLalBA ${ }^{(2)}$
}

(1)Doctora en Geografía

${ }^{(2)}$ Magister en Ciencias Sociales

RESUMEN: Históricamente la necesidad de satisfacer la creciente demanda de energía junto a los progresivos avances tecnológicos en materia de equipamiento energético, han sido causantes de diversas transformaciones territoriales en el mundo. En el siglo XXI, con el fin de compensar los déficits del sistema energético, Argentina apuesta mediante estímulos estatales al desarrollo de proyectos de energía no convencional -renovables y no renovables- imprimiendo un nuevo dinamismo en áreas ricas en recursos como en aquellas capaces de ofrecer los insumos, la infraestructura y los servicios que requiere su aprovechamiento. A través de una metodología mixta de carácter cuanti-cualitativo sobre fuentes documentales y estadísticas, el trabajo busca comprender las transformaciones territoriales que inducen los cambios en las redes energéticas, a partir del estudio del área portuaria de Bahía Blanca-Coronel Rosales. Las ventajas competitivas en infraestructura y servicios portuarios que ofrece el área analizada la convierten en un nodo logístico estratégico frente a las demandas energéticas actuales.

PALABRAS CLAVE: Energías no convencionales; Redes; Transformaciones territoriales; Área portuaria Bahía Blanca-Coronel Rosales.

Recibido: 27.01.2019; Revisado: 15.05.2019

Correo electrónico: clementi.luciana@conicet.gov.ar NºRCID: https://orcid.org/0000-0002-6106-2278;

Correo electrónico: svillalba@fch.unicen.edu.ar Nº ORCID: https://orcid.org/0000-0003-4771-8012

Las autoras agradecen los comentarios y sugerencias realizados por los evaluadores anónimos, que han contribuido a mejorar

y enriquecer el manuscrito original. 


\title{
Territorial transformations through changes in the energy networks in the $21^{\text {st }}$ century. The case of the Bahía Blanca-Coronel Rosales port area (Argentina)
}

\begin{abstract}
Historically, the need to meet the growing demand for energy, coupled with technological advances in the field of energy equipment, has been the cause of various territorial transformations in the world. In the $21^{\text {st }}$ century, in order to compensate for shortfalls in the energy system, Argentina bets on non-conventional energy projects -renewable and non-renewable- by introducing state incentives. This triggers a new dynamism not only in areas where there are resources, but also in those which provide inputs, infrastructure and services required for their use. By means of a mixed methodology of quantitative-qualitative nature, using documentary and statistical sources, this paper seeks to examine territorial transformations induced by changes in energy networks, focusing on the study of the port area of Bahia Blanca-Coronel Rosales. The competitive advantages in port infrastructure and services offered by the analyzed area make it a strategic logistical node against current demands.
\end{abstract}

KEYWORDS: Non-conventional energy; Networks; Territorial transformations; Bahía Blanca-Coronel Rosales port area.

\section{Introducción}

tran sistema energético mundial se encuentra en un proceso de transición, entendiéndose como el paso de un modelo basado en la explotación predominante de hidrocarburos convencionales, a otro en el que las energías renovables tomarian mayor protagonismo. Este proceso de cambio estructural -con impacto en las dimensiones técnicas, económicas, sociales y culturales- viene siendo impulsado por las preocupaciones ante la dependencia de los combustibles fósiles y por el impacto de su combustión en el cambio climático.

Desde fines del siglo $X X$ y principios del $X X I$, Argentina comienza a tomar medidas en pos de diversificar su matriz energética. Legislaciones, programas y proyectos de fomento al desarrollo de energías no convencionales comienzan a trazar el camino para avanzar hacia un sistema menos dependiente y más sostenible. Sucesivas medidas estatales han ido conformando un marco de promoción para el aprovechamiento de fuentes no convencionales renovables y no renovables. Entre los primeros antecedentes de estímulo a las energías renovables se destaca El Plan Alconafta (1980), que fomentó la producción de etanol de caña de azúcar como combustible automotor, el Programa de Uso Racional de la Energía, que planteó la creación de centros de investigación y desarrollo de fuentes renovables (Decreto Nacional №2.247/1985) y la Ley 25.019/1998 Régimen
Nacional de la Energía Eólica y Solar, que declaró el interés y estableció incentivos para estos dos tipos de fuentes. Posteriormente, en la década del 2000, la Ley 26.093/2006 Régimen de Regulación y Promoción para la Producción y Uso Sustentable de Biocombustibles y la 26.334/2007 Régimen de Promoción de la Producción de Bioetanol, activaron la industria de los biocombustibles en el país, a través de incentivos fiscales a la producción y la obligatoriedad de mezclar nafta y gasoil con bioetanol y biodiesel en un $5 \%$.

En paralelo a los avances en materia de biocombustibles, comenzó a conformarse un marco normativo a favor del uso de fuentes renovables para la producción de energía eléctrica. Entre las principales medidas, la Ley 26.190/2006 estableció como meta alcanzar el $8 \%$ de energías renovables en la matriz de energía eléctrica para el 2017, el Programa Generación de Energía Eléctrica a partir de Fuentes Renovables (2009) impulsó la primera licitación para la compra de energía eléctrica proveniente de diversas fuentes renovables, y la Resolución $N^{\circ} 108^{1}$ (2011), autorizó contratos de abastecimiento (PPA) entre el Mercado Eléctrico Mayorista y las ofertas de disponibilidad de generación y energía asociada a fuentes renovables presentadas por Agentes Generadores, Cogeneradores o Autogeneradores.

Recientemente, el Estado está dando nuevas señales para el fomento de las energías renovables a través de estímulos renovados: la Ley 27.191/ 2015 Régimen de Fomento Nacional para el uso

\footnotetext{
1 Derogada por la Resolución N² 202/2016 del Ministerio de Energía y Minería.
} 
de Fuentes Renovables de Energía destinada a la producción de Energía Eléctrica que recupera la meta del $8 \%$ de la matriz nacional de energía eléctrica por fuentes renovables para 2018 y proyecta alcanzar un $20 \%$ para 2025 , la licitación pública RenovAR (Rondas 1, 1.5 y 2) con 147 proyectos adjudicados por un total de $4.466 \mathrm{MW}$, la renegociación de antiguas iniciativas paralizadas (Resolución N²02/2016), la habilitación del Mercado a Término de Energías Renovables (Resolución $\mathrm{N}^{\circ}$ 281/2017) con 44 proyectos eólicos y solares aprobados y la regulación de la generación distribuida de energía renovable integrada a la red eléctrica pública (Ley 27.424/2017).

Los intentos por incorporar nuevas fuentes no convencionales también se manifiestan en la sanción de un marco legislativo favorable a la explotación de recursos no renovables. La Ley 26.741/2012, el Decreto $N^{\circ}$ 929/2013 y la Ley $27.007 / 2014$, además de declarar el interés público nacional por el logro del autoabastecimiento de hidrocarburos y de recuperar el $51 \%$ del patrimonio de la empresa YPF (Yacimientos Petrolíferos Fiscales S. A. $)^{2}$, instrumentan el Régimen de Promoción de Inversión para la Explotación de Hidrocarburos, reglamentando las condiciones para las explotaciones no convencionales.

En este contexto, el trabajo integra avances de dos investigaciones en curso, una doctoral ${ }^{3}$ y otra posdoctoral ${ }^{4}$ que tienen como hilo conductor el desarrollo de energías no convencionales en $\mathrm{Ar}$ gentina. El objetivo es comprender las transformaciones territoriales que inducen los cambios en las redes energéticas, tomando el caso del área portuaria de Bahía Blanca-Coronel Rosales como nodo estratégico en el mapa energético argentino.

En este artículo se proponen las transformaciones territoriales como categoría de análisis para ayudar a desentrañar la realidad y entender los cambios en la estructura socio espacial del área abordada.

«Las transformaciones territoriales implican trascender en el análisis de indicadores económicos y sociales hacia el entendimiento de las relaciones que se dan alrededor de la producción espacial en función del modelo económico impuesto» (Mahecha Ramirez, 2016:119).

A partir de las transformaciones territoriales que es posible encontrar explicaciones que permiten

2 Principal empresa dedicada a la exploración, explotación, destilación, distribución y producción de energía eléctrica, gas, petróleo y derivados de los hidrocarburos en el país.

${ }^{3}$ CONICET, centrada en el análisis de los cambios en las conocer o interpretar de forma más profunda cómo se integra el espacio local al mundo (GudiÑo, 2005). Éstas son entendidas como fenómenos de cambio que se producen en un territorio dado y en un periodo de tiempo determinado, los cuales tienen su manifestación en la forma o morfología urbana, industrial y rural. No obstante, el simple estudio morfológico si bien es necesario como punto de partida del análisis territorial, resulta insuficiente si no se profundiza la interpretación de las relaciones sociales implícitas en ella o el estudio de las fuerzas económicas y sociales que influyen en la configuración territorial otorgando nuevas funciones.

"La forma sólo puede entenderse a través de la función, es decir, lo que se hace o se hizo en el lugar, la naturaleza de actividades predominantes y la categoría de los servicios que se presentan" (GudIÑo, 2005:15).

Dichas transformaciones, a su vez, lejos de presentarse como una acción externa que recibe un territorio para la realización de un proyecto, están ligadas a los procesos en curso, a estrategias de los actores y a las posibilidades para acelerar tendencias preexistentes o asociar nuevos procesos a los territorios (SILVEIRA, 2003).

La investigación se basa en una metodología mixta con un diseño de carácter cuanti-cualitativo, a través de análisis documental y estadístico. La misma se apoya en el empleo de fuentes secundarias (bibliografía específica, informes públicos, artículos periodísticos, entre otros) y primarias, a partir de observación en el terreno y una serie de entrevistas semiestructuradas realizadas a informantes claves durante jornadas de trabajo de campo. La elección del área portuaria de Bahía Blanca-Coronel Rosales como foco de análisis se debe a que representa un nodo clave en las redes energéticas argentinas, ya que en ella convergen recursos provenientes de la Patagonia, e importados -hidrocarburos y electricidad- que sirven al abastecimiento metropolitano y favorecen el fortalecimiento del tejido industrial provincial y regional.

El artículo se estructura en dos partes. La primera describe la impronta territorial del área de estudio en torno al sistema energético nacional, haciendo hincapié en la disponibilidad de recursos, la presencia de actores y el desarrollo de infraestructuras. La segunda, explica las transformaciones territoriales que experimenta el área abordada en

redes y las transformaciones territoriales que se generan a partir de la explotación de hidrocarburos no convencionales. ${ }^{4}$ CONICET, centrada en el análisis de la Energía eólica en Argentina. Transformaciones en las redes y mutaciones territoriales emergentes. 
la última década, a partir de las infraestructuras que se adaptan y las dinámicas que se crean en función de las nuevas demandas y de la planificación de escenarios deseados en materia energética.

\section{2. Área portuaria Bahía Blanca- Coronel Rosales en el marco del sistema energético argentino}

El análisis de este trabajo se centra en una zona de transición entre la región Pampeana y Patagónica. Específicamente el área de estudio comprende los partidos de Bahía Blanca y Coronel Rosales en el Sudoeste de la Provincia de Buenos Aires. Este espacio pertenece al estuario de planicie costera de Bahía Blanca ${ }^{5}$, el cual representa la transición de los ambientes de aguas continentales a los marinos. El canal principal del estuario da acceso al mayor sistema portuario de aguas profundas que viabiliza conexiones con mercados externos, a partir del complejo de puertos de Bahía Blanca (Ingeniero White, Galván, Cuatreros) y Rosales (Perillo \& Piccolo, 2004). Además de estos puertos comerciales el área cuenta con el Puerto Belgrano que oficia como base naval militar. Sobre la franja costera del estuario se emplazan las ciudades de Bahía Blanca (301.572 hab.), Punta Alta (62.000 hab.), Ingeniero White (10.486 hab.) y General Cerri (6.745 hab.), siendo Bahía Blanca la de mayor jerarquía, la cual concentra las principales funciones económicas y administrativas del Sudoeste bonaerense (INSTITUTO NACIONAL DE Estadísticas y Censos, 2010).

La presencia de buenas vías de acceso terrestre en el área, a través de rutas -nacionales y provinciales- y numerosos ramales ferroviarios, permiten conectar gran parte de la región Pampeana y el Norte de la Patagonia. Esto la constituye en un nodo estratégico de conexión y articulación regional de flujos en el país (FIG. 1).

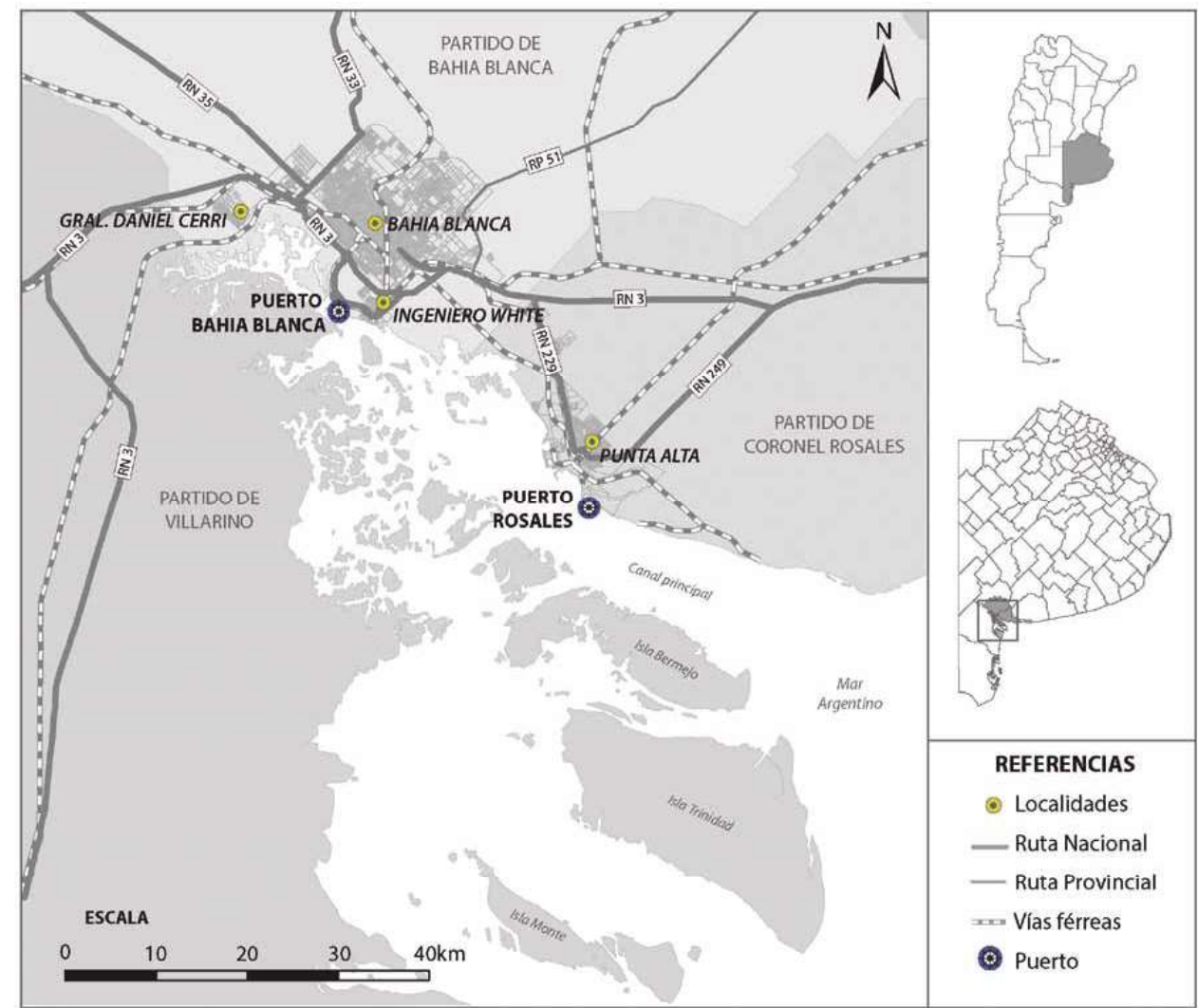

FIG. 1/ Estuario de Bahía Blanca.

Fuente: Elaboración propia basada en información geoespacial del Instituto Geográfico Nacional, 2018

${ }^{5}$ Por su relieve bajo y la presencia de una extensa planicie en forma de embudo elongado. 
A su vez, cuenta con el puerto de aguas profundas de mayor jerarquía a nivel nacional.

Otras de las actividades productivas que más dinamismo imprime en el área, es el complejo o polo petroquímico vinculado a los puertos de Ingeniero White y Galván, creado en el año 1972. Éste cuenta con 3 tipos de industrias: petrolera, petroquímica y química, concentrando el $65 \%$ de la producción petroquímica del país. Entre las firmas más importantes que lo integran se encuentran: PPB Polisur, propiedad de Dow Chemical Company, que produce etileno y polietileno; Compañía Mega -una sociedad anónima cuyos accionistas son YPF S.A, Petrobras y Dow Argentina- que también genera etileno, materia prima de polietilenos y policloruro de vinilo; Profertil S.A. -integrada por Repsol YPF S.A. y Agrium Inc.- que produce urea granulada y amoníaco líquido y Solvay Indupa, que produce policloruro de vinilo. Los derivados de estas materias primas petroquímicas son indispensables para asegurar la disponibilidad, calidad, preservación y confort en comunicaciones, salud, vivienda, vestimenta, alimentación, transporte y en general para casi todos los productos y servicios de la sociedad actual.

\subsection{El complejo portuario y su relevancia para la región}

El complejo portuario de Bahía Blanca encuentra su origen en las últimas décadas del siglo XIX, en el marco de inversiones que por entonces comenzaban a realizar las empresas ferroviarias extranjeras. La particular configuración de la costa, caracterizada por la prolongación de la ría hasta lo profundo de la bahía, internándose entre islas, otorgaba a las aguas la tranquilidad para considerarlas como lugar seguro para las embarcaciones.

La construcción de instalaciones portuarias se inicia en la década de 1880 a raíz de la concesión otorgada por el gobierno nacional a Ferrocarril del Sud. En 1885 se inaugura el muelle de hierro de traza curva, al cual llegaban 5 vías del ferrocarril y 8 grúas, con capacidad de atracar hasta 3 buques de ultramar y otros de cabotaje (TRUJILLO, 2006). A partir de allí, el puerto comercial de Bahía Blanca comenzó a funcionar de manera inmediata y 4 años después, salían por él aproximadamente el $4 \%$ de las exportaciones del país. Progresivamente el movimiento portuario se intensificó de tal manera que su infraestructura resultó insuficiente y requirió la ejecución de nuevas instalaciones como Muelles de Alto Nivel, Muelle de Elevadores y depósitos para ampliar su capacidad de almacenaje. A su vez, desde el sector granelero y agroindustrial se realizaron importantes inversiones en el área portuaria, acorde con el crecimiento en los mercados internacionales, como así también, desde el sector petroquímico, con proyectos en el marco de bloques regionales (Bustos CARA \& TONELLOTTO, 1995).

Actualmente la zona portuaria de Bahía Blanca se compone de un conjunto de instalaciones que abarcan $25 \mathrm{~km}$ de la margen Norte de la ría de la bahía. La jurisdicción portuaria en el ámbito terrestre comprende los puertos de Ingeniero White y Galván como así también la zona aledaña conocida como Cangrejales. La obra más reciente fue la construcción del muelle multipropósito en 2005 , el cual permite una transferencia anual de hasta 200.000 contenedores.

El complejo portuario ofrece una salida directa al Océano Atlántico y representa el único puerto de aguas profundas del país, con muelles para operar buques de hasta 45 pies de calado y todo tipo de mercaderías. No obstante, se especializa en el manejo de 3 grupos principales de cargas: 1-granos y subproductos, 2-combustibles líquidos y gaseosos y 3- productos químicos y petroquímicos, representando un importante centro regional económico, social e institucional. Su área de influencia es amplia, recibiendo granos desde el Sur de Córdoba y Norte y Centro de Buenos Aires, manufacturas de origen agropecuario desde el Norte bonaerense y petróleo desde las cuencas hidrocarburíferas patagónicas. Asimismo, posee relevancia internacional, ya que es único en América Latina en contar con un sistema de control de tráfico radarizado. Su alcance transregional se refleja en flujos que llegan a mercados cada vez más grandes del Cono Sur y otros más distantes (CARRIZO, 2003).

Además, desde 1993 representa el primer puerto autónomo de la Argentina administrado por el Consorcio de Gestión del Puerto de Bahía Blanca (CGPBB), entidad de derecho público no estatal $^{6}$. El tipo de representación que tiene el Ente es de tipo corporativo, es decir, el poder está disperso entre los grupos esenciales del sistema portuario (trabajadores, empresas y Estado).

Ajeno a la órbita del CGPBB, pero dentro del área portuaria analizada, se encuentra el Muelle de

${ }^{6}$ Figura jurídica que permite integrar lo estatal con lo privado. 
Puerto Rosales inaugurado en 1902, gestionado por la Administración Portuaria Bonaerense y la Base Naval Puerto Belgrano, perteneciente a la Armada Argentina, que además de funciones específicamente militares, cuenta con amplia capacidad técnica y de infraestructura para la reparación de buques.

Otro rasgo diferencial del área portuaria de Bahía Blanca y Coronel Rosales es la existencia del régimen franco ${ }^{7}$. Fue en 1999, cuando a través de la Ley Provincial 12.313 (Decreto Reglamentario $\mathrm{N}^{\circ} 2.003 / 2001$ ), se dio inicio a las actividades de la Zona Franca Bahía Blanca-Coronel Rosales (ZFBBCR) como área donde pueden ingresar mercaderías de origen extranjero sin el pago de derechos aduaneros, es decir, con un trato diferencial consistente en la ausencia de ciertos gravámenes, restricciones y plazos de pertenencia ilimitados (GIMÉnEz \& PICARdI De SASTRE, 1999).

En 2007 la ZFBBCR fue adjudicada al concesionario Zona Franca Buenos Aires Sur S.A., conformado por el CGPBB y la Cooperativa de Luz y Fuerza, Industria y Otros Servicios Públicos, Vivienda y Crédito de la ciudad de Punta Alta. La alianza estratégica de estas dos entidades marcó la puesta en marcha en 2012 del Área Operativa I ubicadaen Punta Alta.

La actividad portuaria y el régimen de zona franca se complementan otorgándole al área una particularidad que resulta atractiva para las empresas vinculadas al comercio exterior,

“La complementación entre zona franca y puerto implica beneficios recíprocos. Para la zona fran$\mathrm{ca}$, el puerto es la vía principal para proyectar al mundo sus vínculos comerciales, potenciando su desarrollo. Para el puerto, la zona franca es un elemento diferenciador con respecto a otros complejos" (TRUJILLO, 2006: 80)

\subsection{Impronta local en relación a la energía}

En las últimas décadas el Sudoeste bonaerense, región en la cual se enmarca el área portuaria de Bahía Blanca y Coronel Rosales, ha adquirido un protagonismo relevante en el nuevo contexto energético nacional. Éste no sólo se relaciona a la confluencia de infraestructuras y actores que se vinculan con el sector sino también a la disponibilidad de recursos naturales que adquieren

${ }^{7}$ Equivalente al de Área Franca, actualmente definido por e Código Aduanero (Ley 22.415), al que remite expresamente la Ley 24.331 de Zonas Francas. valor por su capacidad para generar energía.

«...las regiones aptas para su producción ganan un nuevo significado en el inventario de las posibilidades capitalistas» (SANTOS, 1985:42).

En otras palabras, se produce una valorización de la naturaleza ligada a proyectos político-estratégicos que despliegan ciertos actores en el territorio (VAliente \& SchweITZER, 2016).

Respecto de las infraestructuras y actores, en e área confluyen redes de transporte, distribución y comercialización de gas y electricidad, operadas por diversas empresas que resultan puntos nodales claves para el sistema energético nacional (FIG. 2).

En relación al gas, la región posee una impronta de servicios e infraestructura asociada al recurso gracias a la presencia de una planta regasificadora flotante y el nodo de distribución de Transportadora de Gas del Sur (GueRRERO, 2016). La planta regasificadora flotante opera mediante barcos metaneros procedentes de diversos países para abastecer de Gas Natural Licuado (GNL) al país. Desde 2008 esta unidad flotante de regasificación de GNL, a través del buque Excelsior ${ }^{8}$, se ancló en el muelle de la empresa petroquímica Mega S.A. en puerto Galván como medida temporaria para abastecer los picos de la demanda nacional en periodo invernal (FIG. 3).

El nivel máximo de GNL importado fue en el año 2013, con 45 buques, procedentes de países como Trinidad y Tobago, Nigeria, Catar, España, Estados Unidos, entre otros (FIG. 4).

El contrato con Excelerate Energy con vencimiento a diciembre de 2018 no fue renovado, por lo que sólo se continuará con el puerto regasificador ubicado en la ciudad de Escobar (provincia de Buenos Aires), el cual opera desde el 2011. Esta decisión por parte del Estado, está vinculada a la política de impulso al yacimiento Vaca Muerta ${ }^{9}$ y las nuevas posibilidades que se abren a partir de la explotación de hidrocarburos no convencionales en materia de abastecimiento de la demanda interna, la exportación y la generación de divisas para el país

En el Complejo de Procesamiento General Cerri, de la empresa Transportadora de Gas del Sur S.A. (TGS), convergen los gasoductos más importantes del país. Allí se realizan tareas de procesamiento, compresión y mantenimiento; se extraen del gas productos como etano, propano

8 Propiedad de la empresa Excelerate Energy.

${ }^{9}$ Yacimiento que posee las mayores cantidades de recursos técnicamente recuperables del país, estimados por la International Energy Agency. 


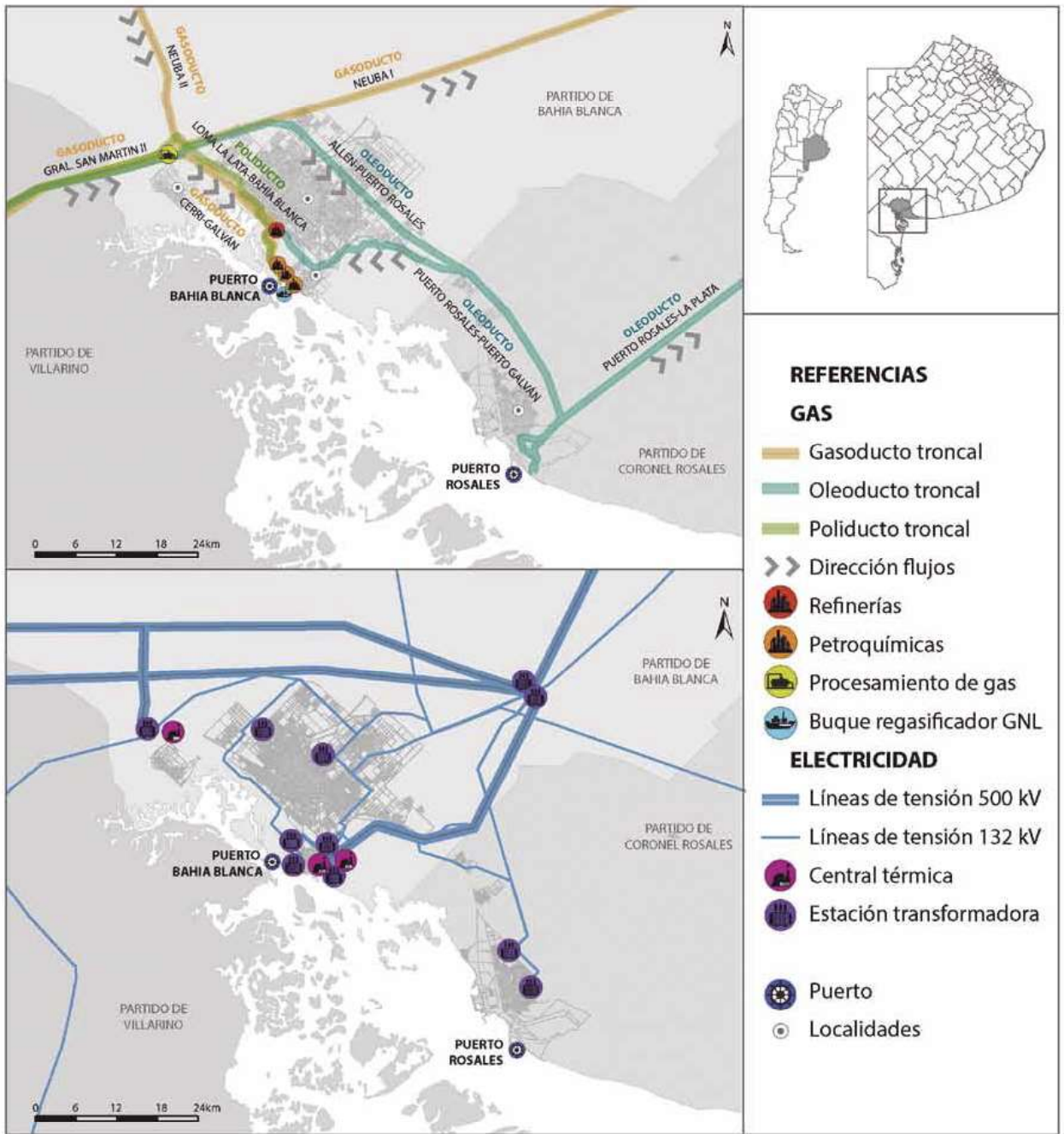

FIG. 2/ Infraestructura vinculada a los servicios de gas y electricidad en el área.

Fuente: Elaboración propia basada en información geoespacial del Instituto Geográfico Nacional y el Ministerio de Energía y Mineria, 2018.

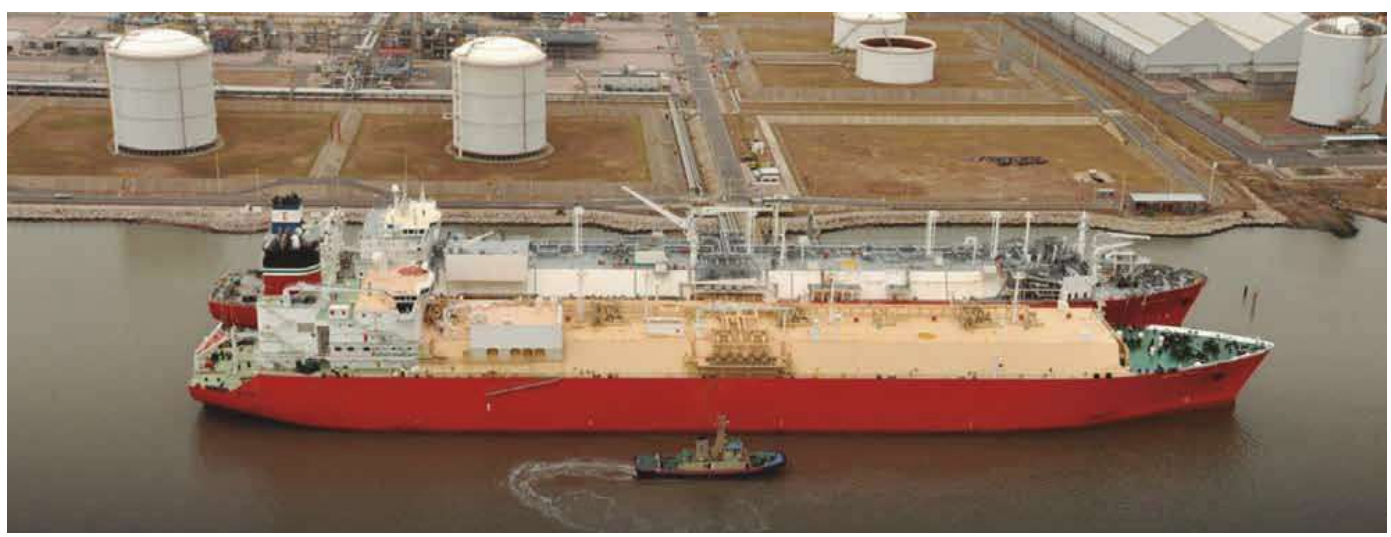

FIG. 3/ Buque Excelsior, unidad flotante de regasificación de Gas Natural Licuado en Puerto Galván.

Fuente: EXCELERATE ENERGY, 2018. 


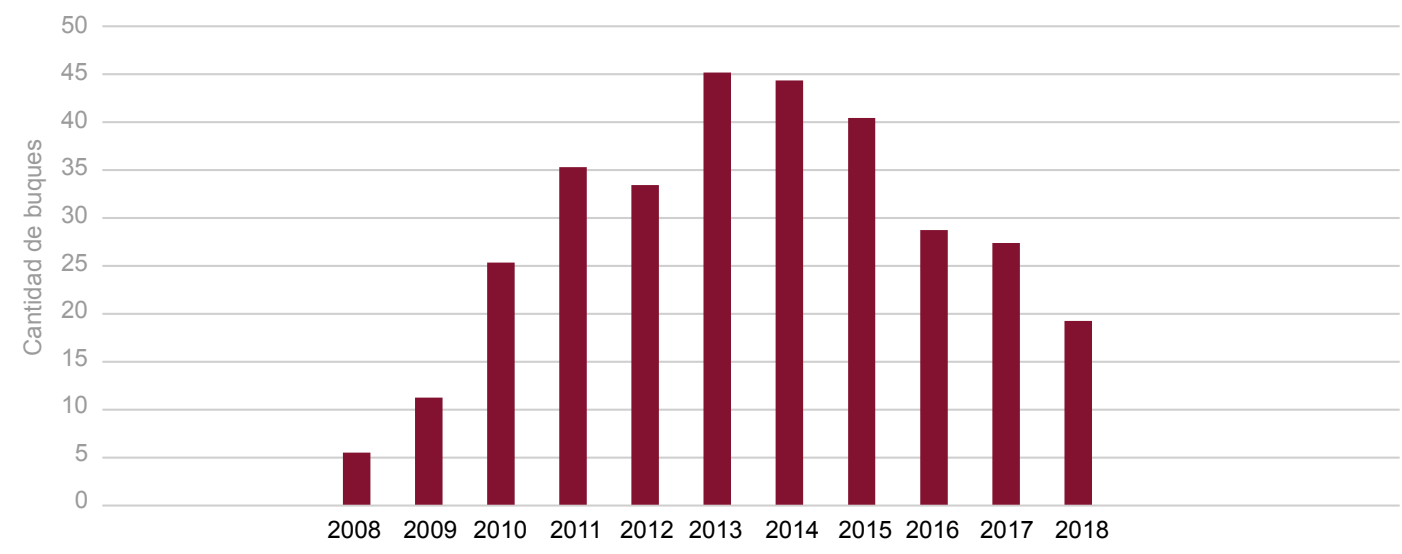

FIG. 4/ Evolución de los buques de GNL ingresados en el puerto de Bahía Blanca entre 2008-2018.

Fuente: Elaboración propia en base a datos del CGPBB y ENARSA

y butano; y al mismo tiempo se genera electricidad para cubrir las demandas del complejo y vender al mercado mayorista nacional. En las instalaciones de la empresa en Puerto Galván, se realizan operaciones de almacenamiento de GLP, recepción y despacho de barcos y camiones. Cuenta con 6 islas de carga de camiones que permiten realizar la carga y descarga de hasta 85 camiones por día aproximadamente. Mediante la operación de las postas 1, 2 y 3 de Puerto Galván, TGS despacha anualmente unos 17 buques de GLP (propios); 14 buques de Gasolina Natural (propios); 25 buques de terceros y operaciones por otros 27 buques (con asistencia y servicios) ${ }^{10}$.

Dos de los gasoductos troncales más importantes del país -construidos durante las décadas de 1970 y 1980- (Neuba I de 1.959 km y Neuba II de $2.104 \mathrm{~km})^{11}$ que conectan la cuenca Neuquina con Buenos Aires, atraviesan la ciudad de Bahía Blanca (RIsUeLo, 2010). Tanto los gasoductos troncales como el gas regasificado en el puerto Galván, permiten la disponibilidad de gas en la ciudad que se dirige principalmente al sector comercial, industrial y residencial, a la generación de electricidad y a la industria petroquímica. Es por eso que algunos autores sostienen que «en cuanto al gas natural, Bahía Blanca es el espacio de convergencia de los gasoductos más grandes de Argentina» (CARRIZO, 2003:334).

En relación con la electricidad, el área de estudio posee infraestructuras tanto para la generación

\footnotetext{
10 Transportadora de Gas del Sur S.A.
}

como para el transporte y distribución. La generación eléctrica se concentra en 3 centrales térmicas operadas por la empresa Pampa Energía: 1- Central Térmica Piedra Buena, ubicada en el puerto de Ingeniero White con $620 \mathrm{MW}$ de potencia instalada, 2- Central Térmica Ingeniero White,ubicada contigua a la primera con 100 MW, y 3- Central de Cogeneración EcoEnergía, ubicada en Bahía Blanca. Mientras las primeras dos funcionan a gas natural o fuel oil, la tercera lo hace a través del aprovechamiento de la energía residual de los gases de escape de 3 turbinas de gas ubicadas en la planta compresora de TGS en el complejo General Cerri.

El área también posee impronta en el aprovechamiento del potencial eólico para la generación de electricidad, a partir de las instalaciones pioneras impulsadas por la Cooperativa Eléctrica de Punta Alta (CEPA) a mediados de la década de 1990 para abastecer sus redes locales. La primera fue puesta en operación en 1995 en Pehuén Có, a partir de un aerogenerador de 0,4 MW que permitía cubrir parte de las demandas de la villa balnearia en temporadas estivales. La segunda, correspondiente al parque eólico Centenario, inaugurado en el año 1998 en la intersección de la Ruta Nacional $N^{\circ} 3$ y la Provincial N ${ }^{\circ} 249$ (a 20 $\mathrm{km}$ de la localidad de Punta Alta). El parque eólico Centenario tenía una capacidad total de 1,8 MW gracias a 3 equipos marca Bonus, adquiridos mediante los beneficios que otorgaban organismos de financiamiento europeos para promocionar la industria de aerogeneradores. 
Ambos emprendimientos eólicos actualmente se encuentran paralizados como consecuencias de diversas dificultades que actuaron como barreras frenando su funcionamiento. El peso de los inconvenientes técnicos y las fallas de operatividad, la extensión del plazo de recuperación de la inversión, la imposibilidad de reparar desperfectos por el costo elevado de los repuestos importados, y más tarde, las restricciones a las importaciones, los precios regulados y el subsidio a la energía convencional, quitaron rentabilidad a la producción eólica desalentando los esfuerzos de la cooperativa eléctrica CEPA por sostener estas iniciativas (Clementi, 2018).

En relación al transporte eléctrico, el área goza de buen acceso a la red eléctrica de transporte en alta tensión (132 kV) y extra alta tensión $(500 \mathrm{kV})$ En el nivel de $132 \mathrm{kV}$ se desarrollan 9 líneas, de las cuales 5 se vinculan al sistema provincial operadas por la empresa TRANSBA. En cuanto a las líneas de extra alta tensión, es atravesada por 4 electroductos de $500 \mathrm{kV}$ que se extienden desde la región Comahue a Buenos Aires. Además, cuenta con la Estación Transformadora Bahía Blanca (ETBB), desde la cual se abastece prácticamente la demanda del Sur bonaerense, y a la cual convergen las líneas de $500 \mathrm{kV}$ "Choele Choe -Bahía Blanca". La Estación Transformadora Choele Choeles relevante porque allí llegan las dos líneas de 500 kV provenientes de las Centrales Hidráulicas El Chocón y Piedra del Águila, y la actual línea Puerto Madryn - Choele Choel, que vincula el Sistema Patagónico (SIP) al Sistema Interconectado Nacional (SIN). Sobre ese mismo nodo de 500 kV aportan al SIN la Central Termoeléctrica Luis Piedra Buena y la Central Termoeléctrica Almirante Guillermo Brown (Alonso \& MONTERO, 2014).

Respecto a la distribución, el área está bajo la administración de la Empresa Distribuidora Energía Sur Sociedad Anónima (EDES S.A), quien es la encargada de la concesión del servicio provincial en el Sudoeste bonaerense junto a 3 entidades cooperativas: la de Punta Alta (24.923 usuarios) en Coronel Rosales y Colonia La Merced (83 usuarios) y Cabildo (1.402 usuarios) en el partido de Bahía Blanca (Vitale \& Álvarez, 2015).

\section{Infraestructuras en transformación por las nuevas demandas del contexto actual}

La relación entre energía y tecnología a lo largo de la historia ha sido la razón de grandes cambios territoriales (REQUeJo LIBERAL, 2011 \& BouIELLE, 2004). Los territorios experimentan procesos de cambios a partir de los efectos socioeconómi-cos que inducen las transformaciones en las redes energéticas (LAURELLI \& JACINTO \& CARRIZO, 2011). Así en las sociedades actuales, la necesidad imperiosa de contar con sistemas capaces de satisfacer las demandas crecientes de energía de forma más diversa, sostenible y distribuida junto a los acelerados avances tecnológicos en materia de equipamiento energético, inducen transformaciones en los territorios.

En Argentina las crecientes demandas energéticas, se expresan en un sistema centralizado, deficitario y en estado crítico. Con el fin de contrarrestar este escenario, las políticas de la última década refuerzan el sistema dependiente de los hidrocarburos fomentando la explotación de hidrocarburos no convencionales en el yacimiento Vaca Muerta en la cuenca Neuquina, a la vez que apoyan el aprovechamiento de energías renovables a través de 200 nuevas iniciativas para la generación eléctrica. En ambos casos, los proyectos de energía no convencionales -renovables y no renovables- atraen inversiones, impulsan actividades y demandan servicios, generando transformaciones no sólo en los sitios propiamente dichos donde se localizan, sino que repercuten en diferentes regiones del territorio nacional.

\subsection{Cambios recientes inducidos por proyectos energéticos}

El Puerto de Bahía Blanca presenta una organización espacial, administrativa y sociopolítica que conjuga requerimientos tradicionales y nuevas funciones y actividades en respuesta a las nuevas demandas internacionales. Esto se ve reflejado en nuevas radicaciones, inversiones y emprendimientos por parte de antiguos y nuevos clientes que refuncionalizan espacios y ocupan nuevos (Tonellotto \& Bustos CARA, 2018). Como conse-cuencia ha cambiado el paisaje portuario y su estructura. Mientras que en 1991 la superficie portuaria era de 240 ha., en la actualidad es de 638 ha. (CGPBB, 2017). Recientemente, los cambios en materia energética han creado la necesidad de incorporar nuevos espacios para el desarrollo de plataformas logísticas, capaces de prestar servicios de cargas especiales relacionadas con proyectos actuales de energía eólica e hidrocarburos no convencionales.

En materia eólica, la incorporación de aproximadamente $3.000 \mathrm{MW}$ de potencia a través de 63 proyectos adjudicados para ser construidos, crea nuevas dinámicas y desafíos. Un gran porcentaje de estos proyectos buscan localizarse en el Sur bonaerense próximos al Partido de Bahía Blanca y sobre la costa atlántica, en ciudades balnearias 


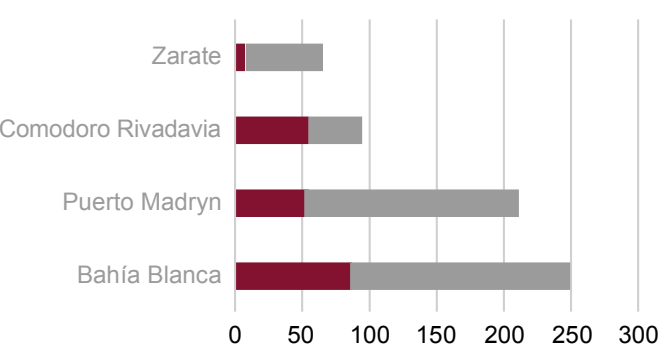

Aerogeneradores ingresados Aerogeneradores por ingresar

FIG. 5/ Ingreso de aerogeneradores según puerto a agosto de 2018.

Fuente: Elaboración propia en base a datos del CGPBB y ENARSA.

como Miramar, Reta o Necochea. Siete de estas iniciativas se circunscriben en el área de análisis de este trabajo. Entre ellos, el proyecto Corti de 100 MW, La Genoveva VI de 86 MW y García del Río III de $10 \mathrm{MW}$ en el marco del programa RenoVAR y el proyecto De la Bahía de $28 \mathrm{MW}$, Wayra I de 50 MW, Pampa Energía de 50 MW y Vientos Bahienses de $48 \mathrm{MW}$ para la venta de energía eléctrica entre privados bajo el nuevo Mercado a Término de Energías Renovables. Entre ellos el proyecto eólico Corti fue el primero en entrar en operación comercial en el mes de mayo de 2018. Éste cuenta con 29 aerogeneradores con una capacidad de producción total de $100 \mathrm{MW}$, localizado sobre una superficie de 1.560 ha. a la vera de la Ruta Provincial $N^{\circ} 51$, a $20 \mathrm{~km}$ al Noreste de Bahía Blanca.

La mayoría de los proyectos eólicos han optado por importar aerogeneradores del orden de los 3 a 3.5 MW de los principales fabricantes del mercado europeo como Vestas y Nordex Group como así también del tecnólogo chino Goldwind. Dichos equipos han comenzado a ingresar al país desde fines del año 2017 a través de los puertos de Bahía Blanca (Buenos Aires), Puerto Madryn (Santa Cruz) y Comodoro Rivadavia (Chubut) principalmente. Hasta agosto del 2018, 204 aerogeneradores ingresaron y otros 418 arribarán entre fines del 2018 y principios de 2019 (FIG. 5).

Más de una tercera parte de ellos arribaron y arribarán al Puerto de Bahía Blanca: 86 ya han ingresado (y parte de ellos ya han sido despachados al punto final) y 163 están por ingresar (FIG. 6).

En relación a la actividad hidrocarburífera, el desarrollo de los recursos no convencionales en la región de Vaca Muerta también ha dinamizado la zona portuaria de Bahía Blanca. La técnica de fracturación hidráulica que se aplica para extraer estos recursos requiere de un fluido de fractura que se compone de agua, aditivos químicos y arena. Este último componente adquiere relevancia porque es el que permite la fluidez del petróleo y gas a la superficie, haciendo que permanezcan abiertas las fisuras realizadas en la formación sedimentaria. En Argentina gran parte de las arenas utilizadas llegan desde el exterior. Sólo la empresa YPF estaría utilizando arenas naturales del país que se extraen de las provincias de Chubut y Entre Ríos (YPF, 2015).

El puerto de Bahía Blanca es una de las puertas de entrada de este producto al país. La evolución en la importación del mismo ha crecido exponencialmente desde 2017. La arena, procedente de países como China y Brasil, arriba al puerto en bolsones dispuestos dentro de contenedores. Uno de los sitios de almacenamiento es el Área Operativa 1 de Coronel Rosales, perteneciente a la Subzona Franca. Luego, esos bolsones son cargados en camiones que se destinan a la región Vaca Muerta (FIG. 7). La logística de este insumo resulta uno de

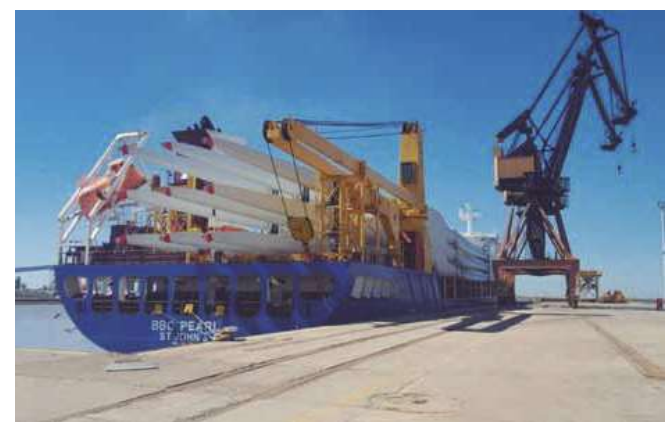

FIG. 6/ Ingreso de aspas de aerogeneradores a través buque el BBC Pearl al muelle multipropósito de Ingeniero White (sitio 21).

Fuente: Sitio Web del CGPBB.

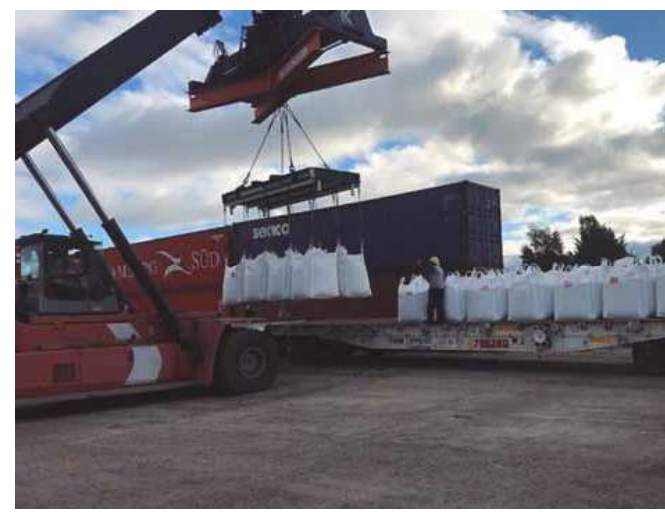

FIG. 7/ Carga de camiones con bolsones en el Puerto de Bahía Blanca.

Fuente: Sitio Web del CGPB. 
los eslabones centrales para reducir los costos de la extracción de hidrocarburos no convencionales.

El dinamismo creado en torno a estos nuevos proyectos energéticos ha dado lugar a la creación de un régimen especial para el almacenamiento de equipamiento: la Subzona Franca Puerto Galván dentro de la Zona Franca Bahía Blanca-Coronel Rosales (Resolución AFIP 4241/2018). Ésta constituye la primera subzona franca del país habilitada con el propósito especial de fomentar el comercio y la actividad industrial exportadora, a través de la reducción de costos y la simplificación de los procedimientos administrativos, en todo aquello relacionado con la importación del material para la producción de energía eólica y explotación de hidrocarburos.

Frente a la competencia de otras terminales marítimas como Zárate y Puerto Madryn, el CGPBB junto con operadores logísticos, la Terminal Patagonia Norte, el Ente Zona Franca y la Concesionaria de Zona Franca, en 2017 han invertido cerca de 40 millones de pesos con el fin de adaptar la infraestructura de arribo y almacenaje del puerto local a las necesidades de carga de proyectos relacionadas con nuevas inversiones productivas en el país, principalmente de sectores como energías renovables e hidrocarburos (FIG. 8). Esta innovación brinda al sistema portuario una ventaja única al poder ofrecer el régimen franco a pie de muelle, con un área de 6 ha. a cielo abierto dedicadas a descarga de proyectos que permiten que el comercio exterior se desarrolle en las condiciones de mayor competitividad global (VICECONTE \& al., 2018).

«Las energías renovables fue un disparador para que como puerto se concreten y fortalezca la creación de grandes áreas logísticas destinadas al depósito y transporte de aerogeneradores y futuros emprendimientos multimodales» (Autoridades del CGPBB, 2018).

El Área de Operación está situada en la Isla Cantarelli de Coronel Rosales y dispone de 2 galpones de $1000 \mathrm{~m}^{2}$ cada uno y una altura de almacenamiento de hasta los $8 \mathrm{~m}$ en un predio de 7 ha. cercadas y con seguridad privada.

A partir de noviembre de 2018, el CGPBB busca atraer inversiones y lograr mayor competitividad internacional a partir de reducir los costos del uso del puerto y aplicar descuentos a la tasa de determinadas cargas. Entre estas cargas, se aplica un $15 \%$ de descuento en la Tasa a las Cargas correspondientes a energías renovables e insumos para el desarrollo de Vaca Muerta. Esto generaría aún más dinamismo en la actividad portuaria como también en las actividades energéticas no convencionales.

\subsection{Nuevas proyecciones \\ tras el efecto Vaca Muerta y la energía eólica}

En los últimos años el área portuaria pasó a tener un protagonismo inesperado por su condición para captar diversos insumos relacionados a proyectos energéticos. Las demandas operativas de Vaca Muerta y de los parques eólicos que se proyectan en los próximos años plantean la necesidad de nuevas infraestructuras y servicios, a la vez que abren nuevas oportunidades.

Ante estas nuevas demandas y dinámicas, el complejo portuario comienza a diagramar una visión al 2040 en la cual aspira entre varios ejes estratégicos a: 1- mejorar las conexiones terrestres entre el complejo portuario-industrial y su entorno a través de accesos locales, circulaciones regionales y corredores nacionales y 2-contribuir en la transición energética, promoviendo un complejo portuario con balance cero de energía y el empleo de combustibles más limpios en las embarcaciones en el marco de estrategias de "Green Shipping", "Green Trucking" y "Green Energy".

La decisión política de impulsar el desarrollo del yacimiento de Vaca Muerta crea la necesidad de varios insumos (arena, minerales, cañerías y accesorios) muchos de ellos provenientes del exterior. Para resolver esa provisión el puerto bahiense se afianza como puerta de acceso. Pero luego esa mercadería necesita ser trasladada a la región Vaca Muerta, para lo cual es menester disponer de un transporte adecuado, seguro y eficiente. La solución técnica y económica más conveniente es el transporte por barco al puerto local y desde allí en ferrocarril hasta el yacimiento.

Conectar Bahía Blanca con Añelo (Neuquén), ciudad que se ha convertido en núcleo de servicios a la producción de hidrocarburos no convencionales, requiere de un trazado férreo que hoy existe parcialmente, con tramos en buen estado y otros muy deteriorados. Por este motivo se busca poner en operatividad 700 kilómetros de vías, lo cual supone una inversión de 570 millones de dólares. A fines de 2018, el Ministerio de Transporte de la Nación busca realizar el llamado a licitación del proyecto Tren Norpatagónico a través del sistema de Participación Público Privado (PPP). Esta estratégica obra incluye el mejoramiento de $374 \mathrm{~km}$, la renovación de $208 \mathrm{~km}$ y la construcción de una nueva traza de $83 \mathrm{~km}$ (FIG. 9). Se estima un plazo de aproximadamente cuatro años para completar la totalidad de estas obras, que para su primer año de operación estaría en condiciones de transportar 1,5 millones de toneladas de carga. Las cargas transportadas no sólo serán en sentido Bahía Blanca-Añelo, sino que 
también se proyecta el transporte de producción regional, como peras y manzanas.

A su vez, el crecimiento sostenido de la explotación de Vaca Muerta tendría un impacto decisivo sobre el futuro de la industria petroquímica en Bahía Blanca cuyo desarrollo depende en gran parte de la calidad y disponibilidad del abastecimiento de gas natural. Se estima que la mayor cantidad de gas que estará disponible para la industria, permitirá la ampliación de las plantas actuales y la radicación de nuevas empresas para responder a una demanda creciente a nivel mundial y del bloque regional Mercosur. Puntualmente, el caso de la empresa Dow ${ }^{12}$ por ejemplo, ya considera la ampliación de sus instalaciones en el Polo Petroquímico local, apuntando a duplicar su producción, con una inversión de entre 3 y 5 mil millones de dólares para 2023.

EI CGPBB experimenta otros de los efectos de la apuesta por los hidrocarburos no convencionales en Vaca Muerta. El fin del contrato del barco regasificador que operaba desde el 2010 en el puerto, marca un punto de inflexión en la dependencia del sistema energético argentino para con las importaciones de gas y las consecuencias en términos económicos que esto implicaba en la balanza de pagos. Recientemente comienza a manejarse la posibilidad de contratar un barco gasificador, es decir, que haga el proceso inverso para la exportación de gas a otros países del mundo. De esta manera se invertirían los flujos comerciales.

Por otra parte, el impulso al desarrollo eólico también comienza a proyectar nuevas inversiones en el área. Desde la Zona Franca Buenos Aires Sur, el CGPBB y la Cooperativa Eléctrica de Punta Alta para el comercio exterior a nivel regional, se plantean como desafío formar un clúster de empresas en energía eólica. Autoridades del consorcio afirman que

«Reconocemos la necesidad de concebir un espacio territorial que conlleve al desarrollo económico e industrial de la ciudad de Bahía Blanca y su zona, a partir de facilitar la creación e instalación de un agrupamiento de empresas para acompañar el proceso de provisión de servicios, materiales y equipos para la puesta en marcha y posterior mantenimiento de los parques eólicos del programa RenovAR».

Ante este desafío, la entidad está trabajando conjuntamente con el Municipio y el Puerto local para atraer tecnólogos de la industria de aerogeneradores para la producción de componentes nacionales en la región. Entre las empresas que han comenzado negociaciones ante la posibilidad de radicar sus plantas de ensamblaje de góndolas y de bujes y de fabricar torres de hormigón para los aerogeneradores en Bahía Blanca se encuentra la firma Nordex Group ${ }^{13}$. De esta manera, la empresa cumpliría con el $35 \%$ de integración local en los equipos, tal como declaró en sus proyectos adjudicados en el marco del RenovAR.

Asimismo, el aprovechamiento del potencial eólico traería nuevas posibilidades de abastecimiento eléctrico ante las demandas crecientes de las industrias del área. Tal es así, que la empresa Profertil ${ }^{14}$ del polo petroquímico e YPF Luz han firmado un convenio en 2018 para la provisión de energía eólica para la operación de la planta de urea granulada de Ingeniero White por 21 años. Con el acuerdo, el $60 \%$ de energía de la planta de Profertil tendrá origen renovable. Esto significa que YPF Luz proveerá $185 \mathrm{GWh}$ por año.

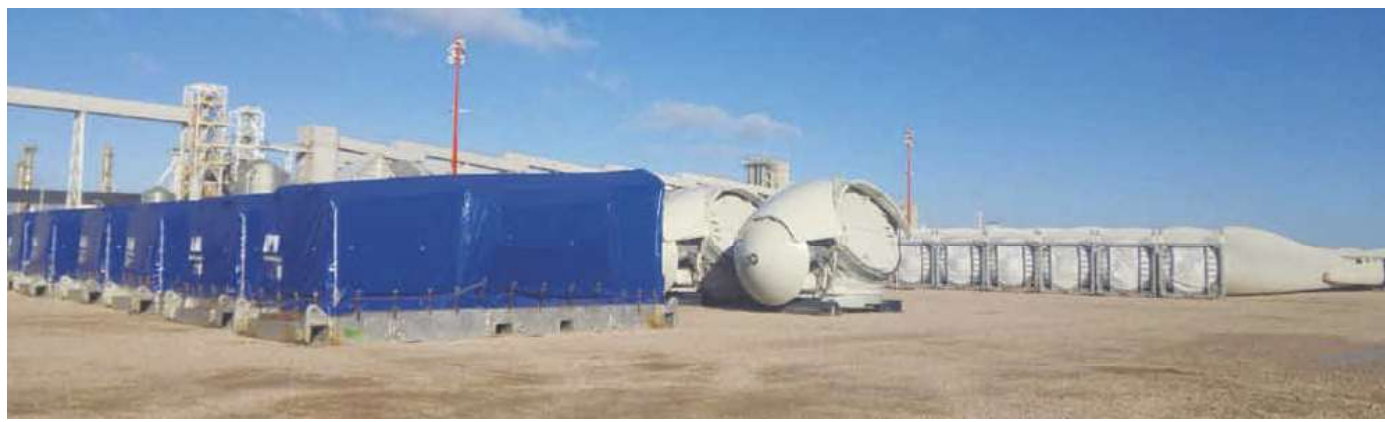

8/ Góndolas, hubs y aspas en el Área Operativa 2 del Puerto Galván (Subzona Franca).

Fuente: Propia, tomada en agosto 2018

${ }^{12}$ El crecimiento de Dow necesita además la adecuación de las instalaciones de TGS y Mega, así como garantizar la provisión de agua.

${ }^{13}$ Fusión entre la compañía Nordex, fundada en 1985 en
Dinamarca y Acciona Windpower, fabricante de generadores eólicos del grupo español Acciona.

${ }^{14}$ La compañía es propiedad $50 \%$ de YPF y $50 \%$ de la canadiense Nutrin. 


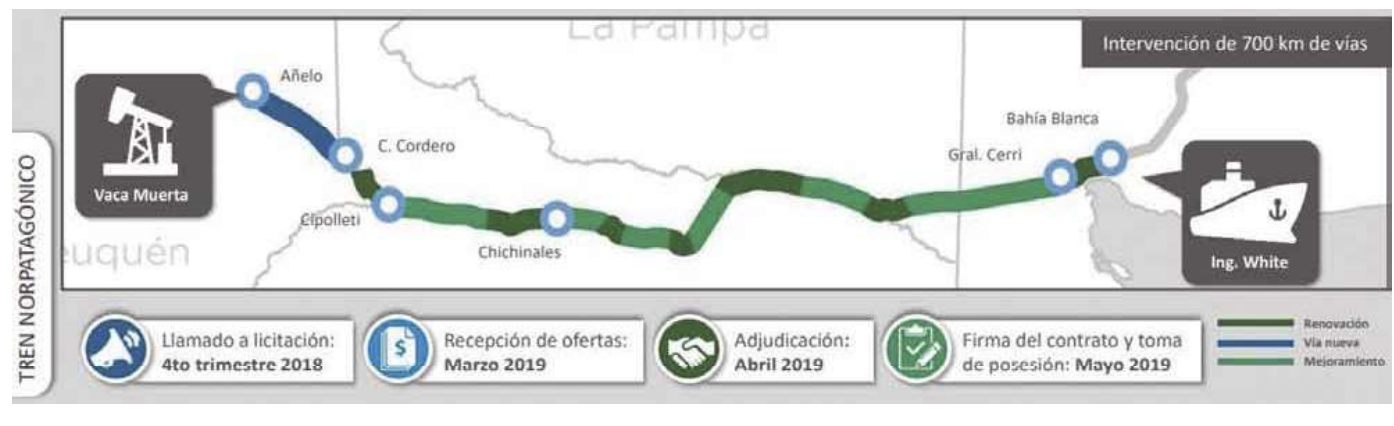

FiG. 9/ Trazado del proyecto Tren Norpatagónico.

Fuente: Ministerio de Transporte, 2018.

\section{Reflexiones finales}

El Sudoeste bonaerense en la cual se enmarca el área portuaria de Bahía Blanca y Coronel Rosales posee una posición estratégica en el entramado de redes energéticas, ya que representa un nodo donde convergen recursos provenientes de la Patagonia y del exterior-hidrocarburos y electricidad-que sirven al abastecimiento metropolitano y favorecen el fortalecimiento del tejido industrial provincial y regional.

Los cambios en el sistema energético nacional de la última década a raíz de la necesidad de asegurar un suministro creciente y menos dependiente avanzan en dos direcciones: el impulso a los hidrocarburos no convencionales y el estímulo a la utilización de fuentes renovables para la producción de electricidad. En ambos casos, los proyectos de energía no convencionales -renovables y no renovables- atraen inversiones, impulsan actividades y demandan servicios, generando transformaciones en los sitios donde se localizan, como así también en diferentes regiones del territorio nacional.

La decisión política sobre explotar el yacimiento Vaca Muerta en la cuenca neuquina y desarrollar nuevos parques eólicos en el Sur bonaerense y la región Patagónica principalmente, implica nuevas demandas de insumos y servicios, lo cual despierta la vocación de ciertas regiones capaces de satisfacerlas. En este caso el área analizada posee ventajas en lo que respecta a la infraestructura y servicios portuarios clave para el ingreso de los insumos y el equipamiento que requieren estos proyectos. Asimismo, goza de un régimen especial para su ingreso y almacenamiento.
Frente a la proyección de escenarios futuros el perfil de nodo logístico competitivo que comienza a adquirir el área podría ser profundizado a partir de obras de infraestructura de transporte vial como el Tren Norpatagónico, corredor clave para la circulación de insumos, e industrial a partir de la radicación de tecnólogos de la industria eólica a través de un clúster o polo eólico.

Paralelamente, el crecimiento sostenido de la explotación de Vaca Muerta como la diversificación de los proyectos eólicos abren nuevas oportunidades para el futuro del polo petroquímico en $\mathrm{Ba}$ hía Blanca, ya que la mayor cantidad de gas y de electricidad que estará disponible para la industria permitirá la ampliación de las plantas actuales y la radicación de nuevas.

El desarrollo de estrategias competitivas mediante la diferenciación de servicios, ha sido un factor de crecimiento e innovación que ha dado impulso a una transformación del área portuaria bahiense desde su origen hasta la actualidad.

\section{Bibliografía ${ }^{15}$}

Agencia Argentina de InVersiones y Comercio InternaCIONAL (INVEST). (2018): Disponible en http://www.inversionycomercio.org.ar/ Fecha de consulta: 2 de octubre de 2018

Alonso, G. \& MonTERo, J. (2014): Impacto de un electroducto "hvdc" sobre el desarrollo de generación eólica de gran porte en Bahía Blanca y la región. I Congreso de Energías Sustentables. Bahía Blanca: Universidad Tecnológica Nacional, p. 159-171.

Bouielle, D. (2004): Manual de Economía de la Energía. Bariloche: IDEE/FB, 275 p.

${ }^{15}$ Las leyes y resoluciones mencionadas en el presente trabajo pueden ser consultadas en el sitio oficial Infoleg: http://www.infoleg.gob.ar 
Bustos Cara, R \& Tonellotto, S. (1995): «Puerto de Bahía Blanca (Buenos Aires, Argentina): Punto de impacto y convergencia de Sistemas de Acción Territorial». http://observatoriogeograficoamericalatina.org.mx/egal6/Geografiasocioeconomica/Ordenamientoterritorial/142.pdf [Consulta: 3 de septiembre de 2018]

CARRIZO, S. (2003): Les hydrocarbures en Argentine. Réseaux. Territoires. Intégration (Thèse de doctorat). Francia: Université Sorbonne Nouvelle Paris 3, $439 \mathrm{p}$.

ClementI, L. (2018): «Energía Eólica y territorios en Argentina. Proyectos en el Sur de la Provincia de Buenos Aires entre fines del siglo XX y principios del siglo XXI» (Tesis doctoral). Bahía Blanca: Departamento de Geografía y Turismo de la Universidad Nacional del Sur, $378 p$

Giménez, M. \& Picardi de Sastre, M. (1999): Zona Franca como instrumento de desarrollo. Bahía Blanca: Editorial de la Universidad Nacional del Sur, $81 \mathrm{p}$.

GudiÑo, M. E. (2005): «Transformaciones territoriales asociadas a la globalización. Una reflexión teóricametodológica». Tiempo y espacio, p. $7-28$.

GueRRERO, A. (2016): La nueva geopolítica de la energía en la región sudamericana. Tendencias, actores y conflictos en la industria del gas. (Tesis doctoral). Bahía Blanca: Universidad Nacional del Sur, 431 p.

Consorcio de Gestión del Puerto de Bahía Blanca (CGPBB). Informe Visión Portuaria 2040. https:/l puertobahiablanca.com/vision portuaria 2040/files/ downloads/Vision BB 2040.pdf

[Consulta: 20 de octubre de 2018]

EXCELERATE ENERgy (2018): Bahía Blanca GasPort. http://excelerateenergy.com/project/bahia-blancagasport/ [Consulta: 5 de septiembre de 2018]

Instituto Nacional de Estadisticas y Censos (2019): Censo Nacional de Población 2010.

https://www.indec.gob.ar/bases-de-datos.asp [Consulta: 28 de septiembre de 2018]

Instituto Geográfico Nacional (2018): Información Geoespa-cial, Capas SIG.

https://Www.lgn.Gob.Ar/Nuestrasactividades/Informaciongeoespacial/Capassig [Consulta: 30 de agosto de 2018]
Laurelli, E. \& Jacinto, G. \& CARRIZO, S. (2011): «Redes energéticas en la Argentina: Planificación territorial en un nuevo contexto regional». Revista de estudios regionales y mercado de trabajo, vol. 7 p. 89-102.

Mahecha RamiRez, C. J. (2016): «Transformaciones territoriales: Una categoría para explicar las dinámicas de conformación territorial». Bitácora 2, p. 113-120.

Ministerio de EnERgía y MineRía (2018): Datos Energía. http://datos.minem.gob.arl [Consulta: 30 de agosto de 2018]

Perillo, G. \& Piccolo, M. C. (2004): «¿Qué es el estuario de Bahía Blanca?» Ciencia Hoy, vol. 14, n 81, p. 55-61.

RequeJo LiBeRAL, J. (2011): «Territorio y energía. Orden mecánico versus orden orgánico». Hábitat y Sociedad, $n^{\circ} 2$, p. 33-47.

Risuelo, F. (2010): «Análisis de la infraestructura de gas natural en la República Argentina. Resumen ejecutivo». Cámara Argentina de la Construcción, Area de Pensamiento Estratégico, $105 \mathrm{p}$.

Santos, M. (1985): Espacio y Método. Sao Pablo: Editorial Nobel, $88 \mathrm{p}$.

Silveira, M. L. (2003): «Por una epistemología geográfica». En Bertonchelo, R. \& Carlos, A. (Eds.) Procesos territoriales en Argentina y Brasil. Buenos Aires: Instituto de Geografía de la Facultad de Filosofía y Letras de la Universidad de Buenos Aires, p. 13-26.

Tonellotto, S. \& Bustos Cara, R. (2018): «El puerto de Bahía Blanca en diferentes contextos políticosideológicos nacionales». Libro de Resúmenes Extendidos. XII Bienal del Coloquio de Transformaciones Territoriales. Bahía Blanca: Asociación de Universidades del Grupo Montevideo, Universidad Nacional del Sur, p. 492-496.

TRANSPORTADORA DE GAS DEL SUR (TGS) (2018): Disponible en http://www.tgs.com.ar/ Fecha de consulta: 2 de octubre.

TRUJILLO, L. (2006): El puerto de Bahía Blanca tras la reforma trujildel Estado: desregulación, descentralización y transformación. Bahía Blanca: Editorial Ediuns, Universidad Nacional del Sur, $90 \mathrm{p}$. 